\title{
A Concept for Electromagnetic Navigated Targeting of Liver Tumors Using an Angiographic Approach
}

\author{
Marius Schwalbe ${ }^{a *}$, Tom Williamson ${ }^{a}$, Iwan Paolucci ${ }^{\mathrm{a}}$, Torsten Fuss ${ }^{\mathrm{b}}$, \\ Iris Baumgartner ${ }^{\mathrm{b}}$, Daniel Candinas ${ }^{\mathrm{c}}$, Stefan Weber ${ }^{\mathrm{a}}$, Pascale Tinguely ${ }^{\mathrm{c}}$ \\ a ARTORG Center for Biomedical Engineering Research, University of Bern, Switzerland; \\ ${ }^{b}$ Division of Clinical and Interventional Angiology, Inselspital, Bern University Hospital, \\ University of Bern, Switzerland; \\ ${ }^{c}$ Department of Visceral Surgery and Medicine, Inselspital, Bern University Hospital, \\ University of Bern, Switzerland
}

\begin{abstract}
Background: The benefits of using navigation technology for percutaneous local ablation of selected hepatocellular carcinoma (HCC) have been shown. Due to additional efforts in the procedural workflow, barriers to introducing navigation systems on a broad clinical level remain high. In this work, initial steps toward a novel concept for simple and precise targeting of HCC are evaluated.

Methods: The proposed technique is based on an angiographic approach using an intrahepatic electromagnetic (EM) reference, for consecutive percutaneous navigated positioning of ablation probes. We evaluated the environmental influence of the angiography suite on EM tracking accuracy, the measurement of a 3D offset from two 2D fluoroscopy images, and the accuracy and efficiency of the proposed approach in a porcine liver model.

Results: The C-arm had a major influence on EM tracking accuracy, with an error up to 3.8 $\mathrm{mm}$. The methodology applied for measurement of a 3D offset from 2D fluoroscopy images was confirmed to be feasible with a mean error of $0.76 \mathrm{~mm}$. In the porcine liver model experiment, the overall target positioning error (TPE) was $2.0 \mathrm{~mm}$ and time for navigated targeting was 17.9 seconds, when using a tracked ablation probe.

Conclusion: The initial methodology of the proposed technique was confirmed to be feasible, introducing a novel concept for simple and precise navigated targeting of HCC.
\end{abstract}

Keywords: Liver Tumor Ablation; EM Navigation; Minimal Invasive; Endovascular Reference; Fluoroscopy

* Corresponding author: marius.schwalbe@artorg.unibe.ch 


\section{Introduction}

Local ablation represents an established low-morbidity and tissue-sparing treatment option for selected patients with hepatocellular carcinoma (HCC) $(1,2)$, inducing local destruction of tumor cells through the delivery of heat by radiofrequency or microwaves. An important and challenging factor influencing the quality of ablative therapy is the adequate intrahepatic localization of tumors and the accurate placement of ablation probes in order to achieve adequate ablation zones (3). When relying on conventional ultrasound guidance, alterations in underlying liver tissue in patients with HCC may render visualization of tumors difficult, and radiation exposure may rise significantly when relying on computed tomography (CT) guidance (4).

To address this challenge and increase precision of liver tumor ablation, image-guidance systems have been introduced with first reports confirming enhanced precision, efficiency and safety of percutaneous navigated ablation of liver tumors (5). Most existing navigation systems rely on the registration of pre- or intra-operative image data to the patient and organ position, a process which might introduce inaccuracies due to displacement of the target relative to the tracked reference marker during or after the registration process. Furthermore, most systems use optical tracking for instrument guidance $(5,6)$, assuming tool rigidity beyond the tracked markers. This potentially leads to tracking errors at the tip of long or thin instruments prone to bending, and renders the tracking of flexible instruments such as angiographic catheters impossible.

To overcome these limitations, electromagnetic (EM) tracking, which is functional through the human body, has been proposed as an alternative $(7,8)$. Due to their small size, EM sensors can be integrated directly in the tip of instruments, avoiding tracking errors due to instrument bending and enabling the tracking of flexible instruments. Nevertheless, accuracy of EM tracking is influenced by ferromagnetic equipment in the proximity of the EM field, which must be evaluated and quantified in each specific clinical setting in order to ensure an optimal navigation environment (7). In the context of liver tumor ablation, various EM-based navigation approaches have been proposed, either using CT based image-to-patient registration by digitizing skin fiducials with an EM tracked pointer $(9,10)$ or using intraoperative imaging in combination with a registration phantom $(11,12)$. To date, various commercial navigation systems utilizing EM tracking for the ablation of liver tumors are available. While some are based on ultrasound imaging (e.g. eTRAX, CIVCO Medical Solutions, Kalona, Iowa, U.S.), others use CT data in combination with a field generator directly placed on the patient (e.g. IMACTIS, La Tronche, France).

Regardless of the applied tracking technology, the interruption of the clinical workflow, due to supplementary procedural steps (registration, calibration), and additional financial efforts represent barriers to introducing navigation technology on a large clinical scale. Although a consistent system design contributes to the reduction of such costs (7), these aspects must be weighed against the expected clinical benefits (safety and reduced recurrence rates due to enhanced precision) and savings due to a more reproducible and potentially faster overall workflow.

To address the challenge of precise targeting of liver tumors while reducing the complexity of the procedure to a minimum, we propose a novel concept for navigated free-hand targeting of HCC. Taking advantage of the benefits of EM tracking and the typical arterial blood supply 
of HCC lesions, we aim to integrate this technology such as to be independent from any explicit registration process and its potential sources of errors, by tracking the tumor at its very origin. In a clinical setting, the proposed technique would imply a transarterial access for placement of an intrahepatic EM reference for consecutive percutaneous navigated free-hand positioning of equally tracked ablation probes.

In this work, we aim to evaluate initial steps towards the proposed technique, by investigating i) the environmental influence of the angiography suite on EM tracking accuracy, ii) the measurement of a 3D offset from two 2D fluoroscopy images, without an explicit registration, and iii) the accuracy and efficiency of the proposed approach in a porcine liver model.

\section{Materials and Methods}

\section{EM tracking tools}

An existing mobile navigation cart (CAS-One Vario, CAScination, Bern, Switzerland) was equipped with EM tracking (Aurora, NDI, Waterloo, Ontario, Canada) and integrated into an angiography suite, containing a ceiling-mounted C-arm (Artis Zee, Siemens, Erlangen, Germany).

A window field generator (WFG, NDI Aurora) with a wide opening $(368 \times 400 \mathrm{~mm})$ and a carbon fiber outer structure was used (13), generating an electromagnetic cylindrical workspace (500 $\mathrm{mm}$ in diameter and $600 \mathrm{~mm}$ height). The WFG was mounted under the angiography table, which equally consists of carbon fiber (Figure 1). The coordinate systems of the EM field generator and the C-arm were aligned by attaching the rectangular WFG parallel to the angiography table with the aid of a water level, to allow a direct relationship between the measurements performed from the angiography images and the coordinates entered into the tracking system.

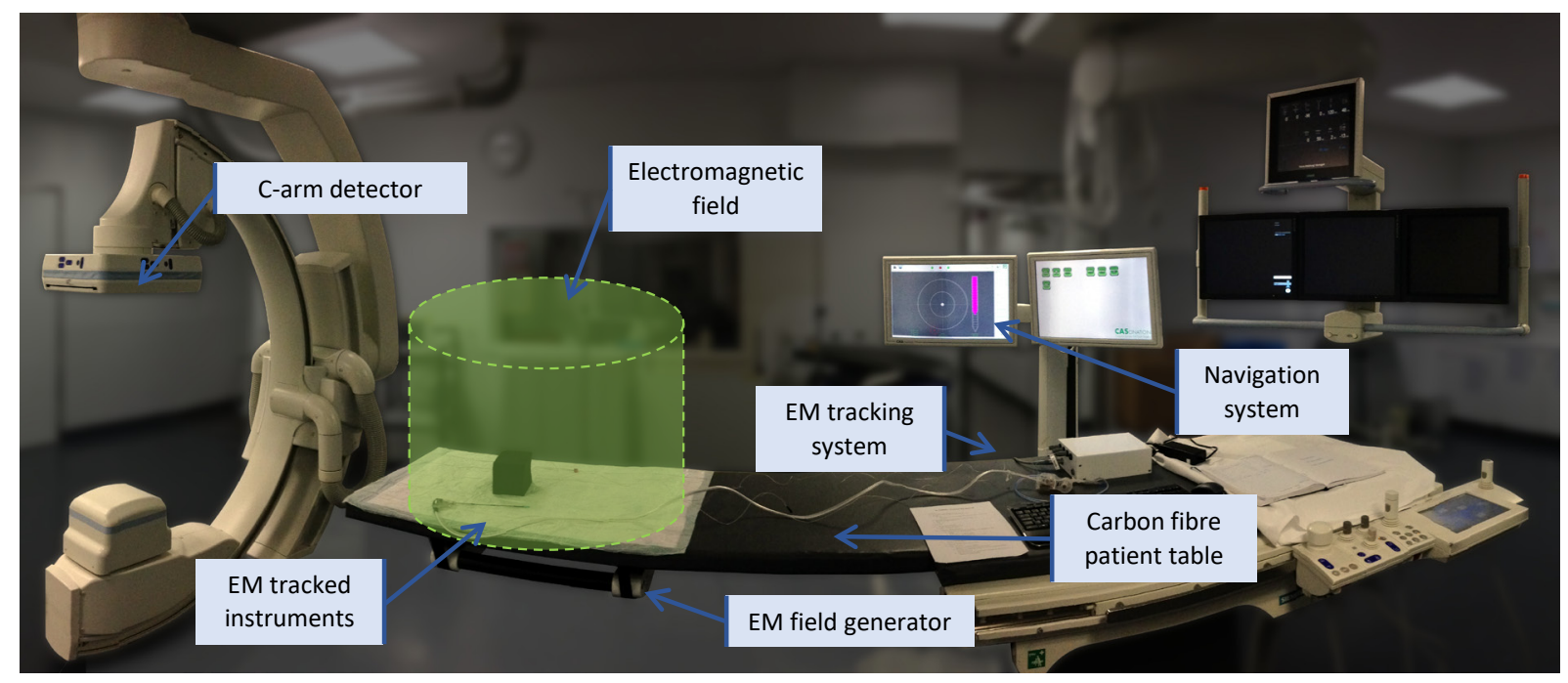

Figure 1. Experimental setup in the angiography suite.

As tracking reference, the smallest available NDI sensor (5DoF, $0.3 \mathrm{~mm} \times 8 \mathrm{~mm}$ ) was integrated into the tip of an angiographic wire. For targeting, a microwave ablation probe (Acculis MTA System, Queensbury, New York, U.S.) was equipped with an EM sensor (NDI, 
5DoF, $0.5 \mathrm{~mm} \times 8 \mathrm{~mm}$ ), which was externally bonded approximately $14 \mathrm{~mm}$ behind the tip of the ablation probe using cyanoacrylate. As an alternative, a 6DoF EM-tracked trocar (Fiagon, Hennigsdorf, Germany) was used for guided insertion of ablation probes without attached EM sensors. The 5DoF EM sensors were aligned with the tools such that the missing $6^{\text {th }}$ DoF aligned with the tool rotational axis.

All tools were pre-calibrated by pivoting, a calibration method which estimates the center of a rotation. To this end, the tools were rotated around the tip and the sensor data were recorded, allowing to calculate the position of the tip (center of rotation) relative to the integrated sensor (14). To reduce jitter and flickering of the tracking signals, the reference sensor position was filtered by a simple moving average filter over the last 20 positions. Since the tracking accuracy varies within the electromagnetic field $(13,15)$, all experiments were conducted in the clinically most feasible region of the field (centered, approximately $10 \mathrm{~cm}$ above the table).

\section{Experimental design and assessments}

\section{Evaluation of environmental influence on EM tracking accuracy}

To quantify the influence of the angiography table and C-arm on EM tracking accuracy, a setup consisting of a fixed configuration of six 5DoF sensors (NDI, $0.5 \mathrm{~mm} \times 8 \mathrm{~mm}$ ) attached to a cube (side lengths $100 \mathrm{~mm}$ ), with two sensors pointing toward each axis of the EM field, was used.

To evaluate the influence of the angiography table, the distances for each pair of sensors (15 distances in total, ranging from $45 \mathrm{~mm}$ to $122 \mathrm{~mm}$ ) were computed in three series of 100 frames as baseline measurements. The same measurements were performed with the setup on the angiography table with the WFG underneath and the entire ceiling-mounted C-arm at maximum distance (190 cm horizontal distance from the center of the WFG). The absolute difference between these measurements to the mean distances of the baseline measurement were calculated and considered as errors.

To evaluate the influence of the $\mathrm{C}$-arm, the same setup was placed in the center of the EM field, and measurements performed with the entire ceiling-mounted $\mathrm{C}$-arm at a maximum distance (190 cm horizontal distance from the center of the WFG) as baseline measurements. The entire $\mathrm{C}$-arm was then moved stepwise towards the field generator in steps of 10 to $20 \mathrm{~cm}$, with measurements at each position.

\section{Evaluation of $3 D$ offset measurement from $2 D$ images}

As the tip of the catheter (i.e. the EM reference) cannot be placed directly in the center of the tumor target in a clinical situation, the measurement of an offset between the actual position of the catheter tip when placed in a tumor-supplying artery and the center of the tumor target becomes necessary (Figure 2(a)). This targeting offset was measured from two orthogonal 2D fluoroscopic images taken at $0^{\circ}$ and $90^{\circ}$, with the $\mathrm{C}$-arm aligned such that the region of interest was positioned on its isocenter (Figure 2(b)).

As the WFG was pre-aligned with the angiography table, the offsets in the images could be measured and directly related to the XYZ coordinates of the tracking system and set in the navigation software. In the $0^{\circ}$ image (Figure 2(b) top), the horizontal distance between tumor center (defined by the center of the circle) and catheter tip corresponds to the X offset, while 
the vertical distance corresponds to the $\mathrm{Y}$ offset. Accordingly, in the $90^{\circ}$ image (Figure 2(b) bottom), the horizontal distance corresponds to the $\mathrm{Z}$ offset and the vertical distance again to the $\mathrm{Y}$ offset. The average of the $\mathrm{Y}$ offsets from both images was used if these values differed. To allow this direct accordance, the acquisition of the images at exactly $0^{\circ}$ and $90^{\circ}$ is essential.
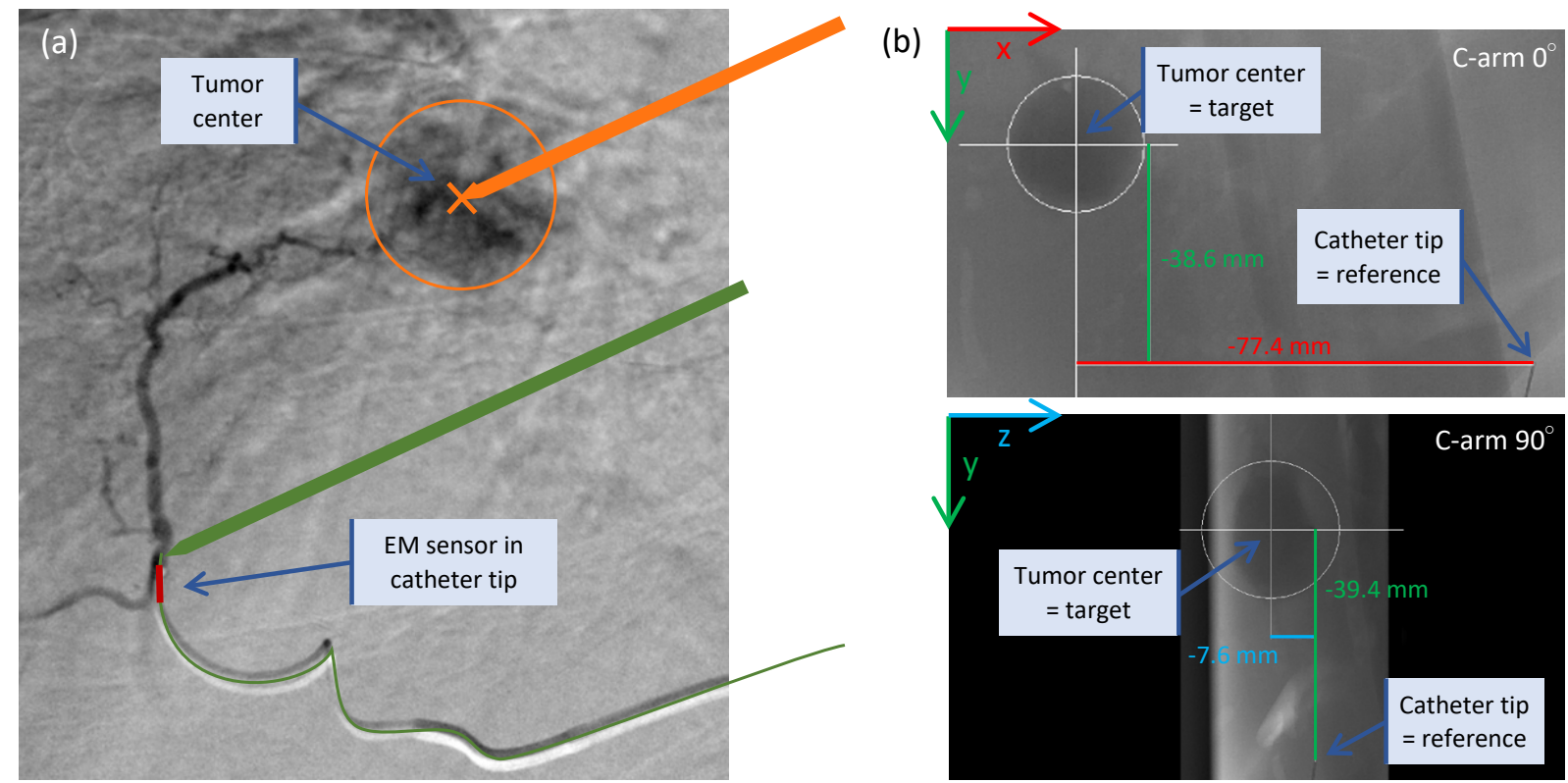

Figure 2. (a) Schematic illustration indicating a microcatheter in tumor-supplying artery of HCC. Overlaid are ablation probes for baseline targeting (green) and offset targeting (orange). (b) Measurement of the XYZ offset between the reference (tip of the catheter) and the target (center of the tumor) in $0^{\circ}$ and $90^{\circ}$ fluoroscopic images.

To evaluate the accordance of the 3D offset measurements with the measurements from the EM system, two 5DoF EM sensors were placed in a cubic foam block (side lengths $140 \mathrm{~mm}$ ) at fixed distances and placed onto the angiography table. Offset measurements from the images between the two sensors were then compared to the direct EM system measurement. To avoid any influence from the $\mathrm{C}$-arm on the EM measurements, the entire ceiling-mounted C-arm was again moved to the maximum distance $(190 \mathrm{~cm})$ from the area of EM measurement.

\section{Evaluation of targeting accuracy in porcine liver model}

For navigated targeting, the software uses the tracking information from two tools: the position of the catheter tip considered as the reference, and the position and orientation of a tracked ablation probe as the targeting device. It displays the relative 3D displacement of the probe with respect to the chosen target, with the information separated into orientation and distance. The orientation represented in a crosshair indicates the lateral distance and a depth bar the longitudinal distance (Figure 3(a)).

The differential position of the probe and the tumor target as displayed on the navigation system is computed as

$$
T_{\text {Tumor }}^{\text {Probe }}=-T_{\text {Catheter }}^{\text {Tumor }}-T^{\text {Catheter }}+T^{\text {Probe }}
$$

where $T_{\text {Catheter }}^{\text {Tumor }}$ is the $3 \mathrm{D}$ offset measured from the two $2 \mathrm{D}$ fluoroscopy images, $T^{\text {Catheter }}$ the 
position of the tracked catheter tip and $T^{\text {Probe }}$ the position and orientation of the tracked ablation probe, the latter two values as measured by the EM tracking (Figure 3(b)).
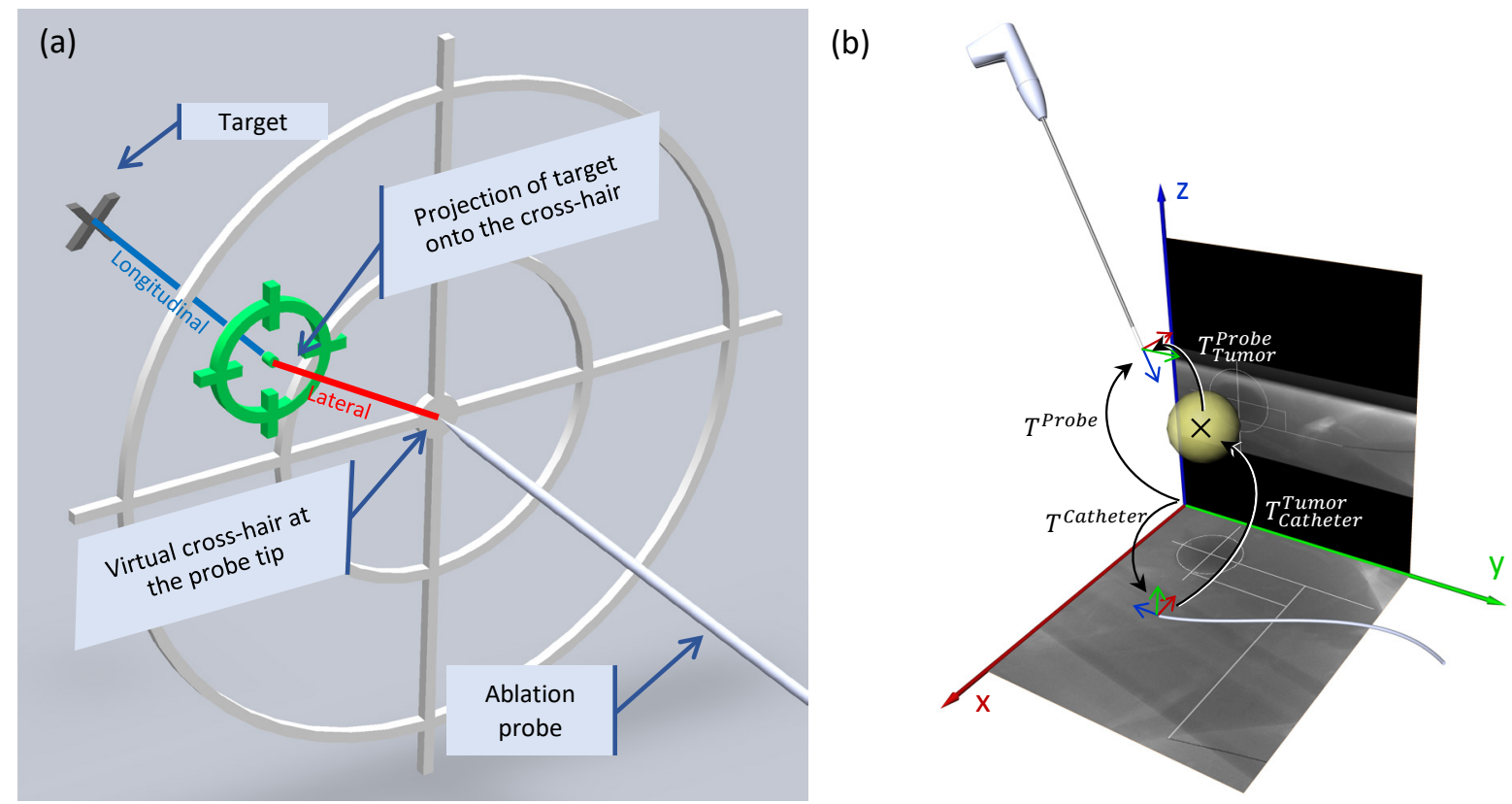

Figure 3. (a) Concept of a crosshair viewer with longitudinal and lateral distances, and (b) transformations involved for the offset targeting.

For the experimental model, artificial tumors (15 to $20 \mathrm{~mm}$ in diameter) consisting of agarose and contrast agent, creating a soft consistency similar to the surrounding liver parenchyma (16), as well as the reference EM sensor were placed between the liver lobes of an ex vivo porcine liver. This model was placed inside a closed torso and positioned on the angiography table (Figure 4(a,b)). Targeting of artificial tumors was performed free-hand under continuous visual guidance by the navigation system, in two different scenarios (Figure 2(a)). To evaluate technical accuracy of the navigation system in the proposed angiographic setting without any additional steps such as offset measurement and imaging involved, the tip of the EM reference was directly targeted (referred to as baseline targeting). To assess accuracy of the technique in a clinically relevant setting with an offset between the catheter tip and the tumor, the center of the tumor was targeted (referred to as offset targeting).

Both baseline and offset targeting attempts were performed using i) the ablation probe with externally bonded EM sensor, and ii) an ablation probe without attached EM sensor inserted through an EM-tracked trocar (Figure 4(c)). As the ablation probe without attached EM sensor allowed only indirect depth control, the distance between the tracked trocar and the target as displayed by the EM navigation system was manually controlled by visual inspection of the marks on the probe. The entire ceiling-mounted $\mathrm{C}$-arm was moved at a maximum distance $(190 \mathrm{~cm})$ from the area of interest during the navigated targeting attempts, to avoid any influence from the $\mathrm{C}$-arm onto the EM tracking accuracy.

Once the ablation probe was positioned, targeting accuracy was assessed as target positioning error (TPE), expressing the Euclidean distance between the defined target and the tip of the targeting tool (17) as measured in the fluoroscopy images. Additionally, time for 
navigated targeting was manually assessed using a stopwatch, measuring time from insertion of the ablation probe to reaching the final position.
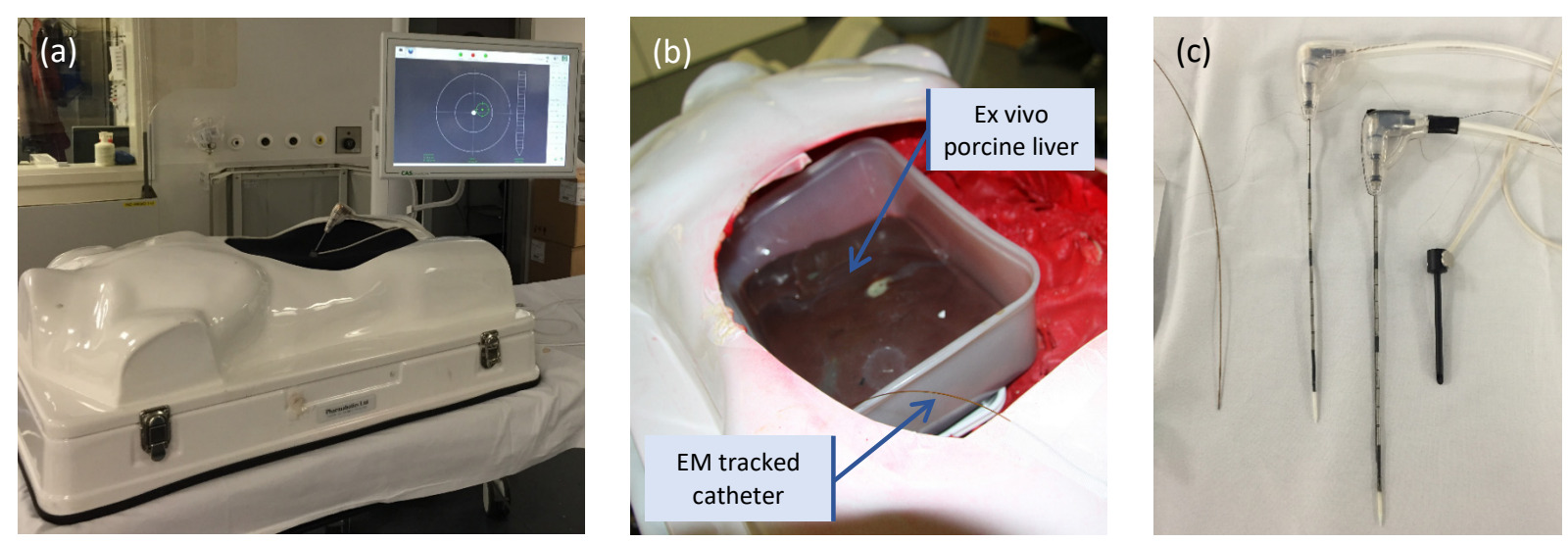

Figure 4. (a) Set-up of the closed torso and the navigation system, (b) the porcine liver inside the open torso, (c) EM tracked instruments (from left to right): EM-tracked catheter used as reference, ablation probe without attached EM sensor, ablation probe with externally bonded EM sensor, EM tracked trocar.

\section{Data analysis}

The TPE in the fluoroscopic images were measured in OsiriX (Pixmeo, Bernex, Switzerland) and processed using Matlab (MathWorks, Natick, Massachusetts, U.S.). Statistical analyses were performed applying the two-tailed, two-sample t-test with a significance level of $\alpha=0.05$, using Prism (GraphPad Software, Inc., La Jolla, California, U.S.).

\section{Results}

\section{Evaluation of environmental influence on EM tracking accuracy}

The influence of the angiography table resulted in a mean error of $0.77 \mathrm{~mm}$ when compared to baseline measurements ( $95 \%$ confidence interval ( $\left.\mathrm{CI}_{95}\right) 0.64-0.90 \mathrm{~mm}, \mathrm{n}=3 \times 15=45$ ).

The influence of the $\mathrm{C}$-arm showed tracking errors starting to increase at a distance of $110 \mathrm{~cm}$ and reaching a maximum of $3.8 \mathrm{~mm}$ when the detector of the $\mathrm{C}$-arm is above the WFG (Figure 5). Errors were strongly dependent on the orientation of the sensors, with the two sensors oriented in $\mathrm{Z}$ direction showing the largest changes in position.

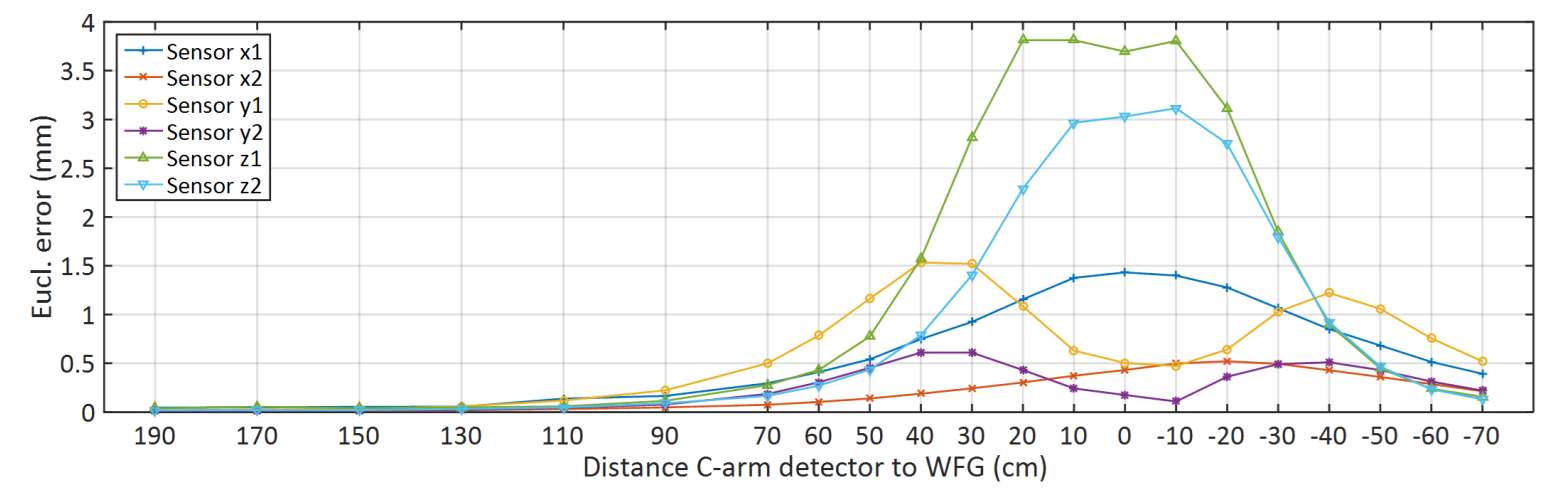

Figure 5. Influence of the C-arm distance on EM tracking errors. The distance of $0 \mathrm{~cm}$ indicates the C-arm centered over the EM field generator. 


\section{Evaluation of $3 D$ offset measurement from $2 D$ images}

In total, five measurements were performed with the two EM sensors at different distances from each other (ranging from $9 \mathrm{~mm}$ to $100 \mathrm{~mm}$ ). Median values for the $\mathrm{X}$ and $\mathrm{Y}$ differences $(0.31 \mathrm{~mm}$ and $0.23 \mathrm{~mm})$ were lower than those from the $\mathrm{Z}$ differences $(0.59 \mathrm{~mm})$, with the Euclidean differences $(0.76 \mathrm{~mm}$, interquartile range $0.49 \mathrm{~mm})$ subsequently dominated by this $\mathrm{Z}$ error component.

\section{Evaluation of targeting accuracy in porcine liver model}

Twelve baseline and twelve offset targeting attempts (offset of $47 \mathrm{~mm}$, Euclidean distance) were performed using the EM tracked ablation probe. Additionally, twelve baseline and twenty-four offset targeting attempts (twelve each with an offset of $46 \mathrm{~mm}$ and $92 \mathrm{~mm}$, Euclidean distances) were performed using the ablation probe without attached EM sensor with the EM-tracked trocar.

Figure 6(a) summarizes TPEs grouped by targeting type (baseline vs. offset) and by targeting modality (ablation probe vs. trocar). The mean TPE using the ablation probe was lower for the baseline attempts $\left(1.7 \mathrm{~mm}, \mathrm{CI}_{95} 0.9-2.6 \mathrm{~mm}\right)$ compared to the offset attempts (2.2 mm, CI $\mathrm{C}_{95}$ 1.4-3.0 mm). A similar trend was observed using the trocar with a mean TPE of $2.2 \mathrm{~mm}\left(\mathrm{CI}_{95} 1.0-3.3 \mathrm{~mm}\right.$ ) for baseline and $2.7 \mathrm{~mm}\left(\mathrm{CI}_{95} 2.0-3.3 \mathrm{~mm}\right)$ for offset attempts. Moreover, the targeting attempts using the tracked trocar were less accurate compared to the attempts using the ablation probe, particularly when performing offset targeting $(2.7 \mathrm{~mm}$ and $2.2 \mathrm{~mm}$ respectively). None of the differences between the various groups were statistically significant, and no relationship was observed between the two different offsets (46 $\mathrm{mm}$ and $92 \mathrm{~mm}$ ) and the resulting mean TPEs for the offset attempts using the tracked trocar (both $2.7 \mathrm{~mm}, \mathrm{n}=12 \mathrm{each}$ ). Two exemplary attempts of baseline and offset targeting are shown in Figure 6(b, c).

Mean targeting time for the targeting attempts using the EM tracked ablation probe was 17.9 seconds ( $\mathrm{CI}_{95} 14.1-21.6$ seconds, range $5-43^{\circ}$ seconds), while targeting times for attempts using the tracked trocar were significantly longer (mean 27.4 seconds, $\mathrm{CI}_{95} 24.1-$ 30.7 seconds, range $15-58$ seconds) $(\mathrm{p}<0.001)$.

(a)

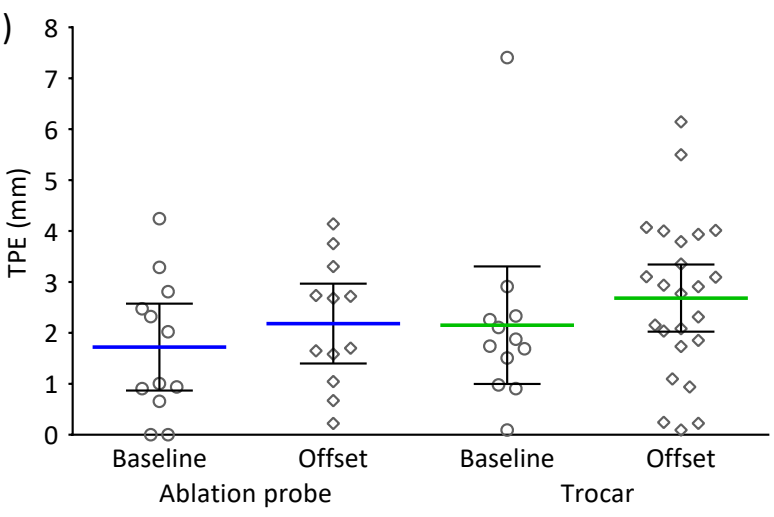

(b)

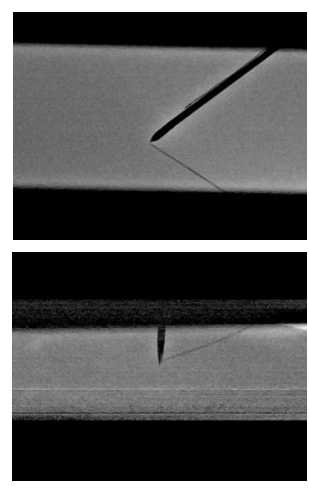

(c)
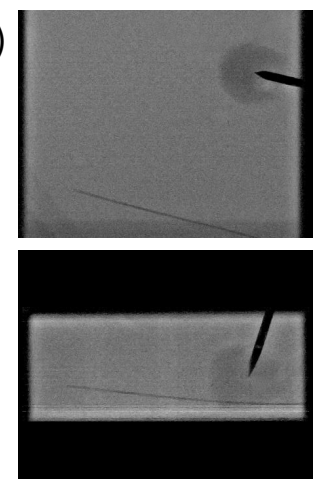

Figure 6. (a) Summary of the target positioning errors (TPE) from all 60 targeting attempts. Means and 95\% confidence intervals are shown. (b) Baseline and (c) offset targeting attempts using the ablation probe with externally bonded EM sensor. 


\section{Discussion}

In this work, we present initial steps toward a novel technique for precise ablation of liver tumors, implying an angiographic approach for endovascular placement of an EM reference sensor and consecutive percutaneous navigated tumor targeting. Methodological feasibility, positional accuracy and procedural efficiency of the technique were confirmed in a series of ex vivo experiments.

First analyses assessed the influence of involved objects within the angiography suite on EM tracking accuracy and thus the functionality of EM tracking within the desired clinical environment. The influence of the angiography table was negligible, however, a significant influence of the $\mathrm{C}$-arm on tracking accuracy was observed, with an increasing measurement error at distances below $110 \mathrm{~cm}$ between the C-arm and the WFG. Hence, all following EM measurements were performed with the entire ceiling-mounted $\mathrm{C}$-arm at maximum distance to minimize the influence on tracking accuracy.

The small differences between 3D offset measurements from 2D images and EM tracking $(0.76 \mathrm{~mm})$ confirm the feasibility of the proposed methodology of measuring a $3 \mathrm{D}$ offset from two 2D fluoroscopic images. This finding is further underlined when comparing the accuracy of the baseline and offset targeting attempts in the porcine liver model. Baseline targeting aimed to assess the basic performance of the proposed technique when using the EM tracking system for targeting in a "best case scenario", without an additional offset measurement. This allowed to gain knowledge about the specific influence of the proposed 3D offset measurement on the targeting error. Accordingly, the difference of $0.5 \mathrm{~mm}$ between the average TPE from baseline and offset targeting confirmed a minimal influence of the proposed offset measurement technique on targeting accuracy. Most importantly, the proposed method of 3D offset measurement allows for navigated targeting of the tumor center relying only on intraoperative imaging. Hence, a possible loss of targeting accuracy due to registration errors can be avoided, the latter representing a frequent and relevant limitation of registration-based navigation $(9,10)$.

The above evaluations implicitly confirm the correct mounting of the WFG under the angiography table, since a misalignment would lead to higher differences between 3D offset measurements from 2D images and EM tracking. Furthermore, no relevant occlusions due to the field generator occurred in the images, as the WFG is explicitly constructed for the use in an angiographic environment. However, low levels of random white noise were observed in the $0^{\circ} \mathrm{C}$-arm images, when the EM field generator was switched on. Nevertheless, no image distortion was observed, and the needles and catheters were clearly visible.

In all experiments, the average targeting accuracy (measured as TPE) was below $3 \mathrm{~mm}$. Although this obviously needs to be confirmed in vivo, this accuracy would lie within a favorable and acceptable range for targeting and ablation of HCC, when aiming at an additional safety margin of 5-10 $\mathrm{mm}(3,18,19)$. In another work, targeting errors of $6.4 \pm 3.3 \mathrm{~mm}(\mathrm{n}=76)$ and $8.3 \pm 3.7 \mathrm{~mm}(\mathrm{n}=32)$ have been reported in phantom experiments and in vivo, respectively (9). Levy et al. applied CT-based image-to-patient registration by digitizing skin fiducials using an EM tracked pointer, with an additional EM tracked intrahepatic needle serving as reference to detect organ motion, and propose the use of multiple internal fiducials to further enhance the accuracy of the system (10). In our proposed technique, an EM tracked reference is placed in immediate proximity to the intrahepatic 
tumor target, thus allowing a synchronous motion of both the tracked reference and target, allowing navigated targeting without an explicit registration.

As expected, the use of a directly tracked ablation probe resulted in higher targeting accuracy than the combination of an ablation probe without attached EM sensor inserted through a tracked trocar. This is likely due to unintended instrument bending as well as the more complex handling of the tracked trocar due to indirect depth control. This confirms that direct tracking of the ablation probe close to the tip of the targeting instrument allowed enhanced targeting accuracy, as has been previously described (20).

The proposed targeting procedure was efficient with an average targeting time of 17.9 seconds ( $\mathrm{CI}_{95}$ 14.1-21.6 seconds) in the porcine liver experiment. Although the time needed for offset measurement itself was not explicitly assessed, metadata of the fluoroscopic images indicate a duration of around 5 minutes. This offset measurement could be easily accelerated using a more dedicated software alternatively to the $\mathrm{C}$-arm console used for our experiments.

To adapt the proposed approach toward an eventual clinical application, the instrumentation applied in this work would need further development. For the intrahepatic placement of an endovascular reference, an EM-tracked angiographic wire adapted to the dimensions of a clinically applicable microcatheter as used for hepatic interventions (e.g. 2.7 French) would be required. While various attempts to integrate EM sensors into angiographic catheters and guidewires have been presented (12), no EM-tracked wire with the required dimensions is currently available. To this end, a prototype of an EM-tracked angiographic wire is currently developed by our team. Furthermore, to the best of our knowledge no ablation probes with directly integrated EM sensors are available to date, although clinical tests with externally bonded sensors have been conducted previously (21).

Next to these more technical elements, various workflow related issues need further investigation towards the integration of the proposed method into a clinical application. Although all experiments were conducted in the relevant region of the EM field (centered, approximately $10 \mathrm{~cm}$ above the table), higher inaccuracies might occur if the patient cannot be positioned as anticipated, since the accuracy within the EM field is not homogeneous $(13,15)$. To confirm the accurate needle placement and to detect a potential dislocation of the reference sensor between offset measurement and targeting, a control image would have to be acquired between needle placement and the actual treatment. Furthermore, as the proposed workflow is focused on a directly tumor-targeted approach using dynamic tracking of the tumor at its origin, additional path planning along the needle trajectory between skin incision and liver would become necessary in a clinical application. In the presented 2D angiographic setting, ultrasound guidance would be a simple and previously described method (22); alternatively the use of cone beam CT could potentially optimize path planning as well as offset measurement for the proposed technique (23).

In a clinical setting, the proposed transarterial approach would obviously represent an additional invasive access for percutaneous ablation, which could however be beneficial when applied in a combined approach of ablation and transarterial chemo-embolization (TACE), where selective catheterization of the tumor-supplying arteries is already performed. Such a combined approach has been shown benefits regarding oncological outcome in selected patients with HCC (24-26). Moreover, the proposed principle (i.e. placing an EM-tracked reference in the immediate vicinity of the target structure to bring an EM-tracked instrument 
into this target structure) represents a generic electromagnetic navigation approach, which might be beneficial in various other medical applications. Since we demonstrated that $2 \mathrm{D}$ fluoroscopy is a simple imaging modality leading to accurate targeting within the proposed technique, this could also be applied in other domains where mostly mobile $\mathrm{C}$-arms are available, e.g. in OR-based fluoroscopy.

\section{Conclusion}

The initial methodology of a novel concept for targeting and ablation of liver tumors using EM navigation in an angiographic setting was demonstrated, allowing precise and efficient targeting in a porcine liver model without the need for an explicit registration process. These initial results will allow the further evaluation of the proposed technique toward a clinical application in vivo.

\section{Acknowledgments}

The authors would like to acknowledge Denise Baumann, Huanxiang Lu, and Juan Ansó for support.

\section{Disclosure statement}

The authors report no conflicts of interest.

\section{Funding}

This work was partially funded by the Ruth \& Arthur Scherbarth Stiftung.

\section{References}

1. Lencioni R, Crocetti L. Local-regional treatment of hepatocellular carcinoma. Radiology. 2012 Jan;262(1):43-58.

2. Bruix J, Gores GJ, Mazzaferro V. Hepatocellular carcinoma: clinical frontiers and perspectives. Gut. 2014 May;63(5):844-55.

3. Bale R, Widmann G, Stoffner DIR. Stereotaxy: breaking the limits of current radiofrequency ablation techniques. Eur J Radiol. 2010 Jul;75(1):32-6.

4. Beyer LP, Pregler B, Nießen C, Schicho A, Haimerl M, Jung EM, et al. Stereotactically-navigated percutaneous Irreversible Electroporation (IRE) compared to conventional IRE: a prospective trial. PeerJ. 2016;4:e2277.

5. Engstrand J, Toporek G, Harbut P, Jonas E, Nilsson H, Freedman J. Stereotactic CTGuided Percutaneous Microwave Ablation of Liver Tumors With the Use of HighFrequency Jet Ventilation: An Accuracy and Procedural Safety Study. AJR Am J Roentgenol. 2017 Jan 20;208(1):193-200.

6. Wallach D, Toporek G, Weber S, Bale R, Widmann G. Comparison of freehand- 
navigated and aiming device-navigated targeting of liver lesions. Int J Med Robot Comput Assist Surg. 2014 Mar;10(1):35-43.

7. Franz AM, Haidegger T, Birkfellner W, Cleary K, Peters TM, Maier-Hein L. Electromagnetic tracking in medicine -A review of technology, validation, and applications. IEEE Trans Med Imaging. 2014;33(8):1702-25.

8. Moncharmont L, Moreau-Gaudry A, Medici M, Bricault I. Phantom evaluation of a navigation system for out-of-plane CT-guided puncture. Diagn Interv Imaging. 2015 Jun;96(6):531-6.

9. Banovac F, Tang J, Xu S, Lindisch D, Chung HY, Levy EB, et al. Precision targeting of liver lesions using a novel electromagnetic navigation device in physiologic phantom and swine. Med Phys. 2005;32(8):2698-705.

10. Levy EB, Tang J, Lindisch D, Glossop N, Banovac F, Cleary K. Implementation of an Electromagnetic Tracking System for Accurate Intrahepatic Puncture Needle Guidance: Accuracy Results in an In Vitro Model. Acad Radiol. 2007;14(3):344-54.

11. Nagel M, Hoheisel M, Petzold R, Kalender W a., Krause UHW. Needle and catheter navigation using electromagnetic tracking for computer-assisted C-arm CT interventions. In: Cleary KR, Miga MI, editors. Proceedings of SPIE. 2007.

12. Condino S, Ferrari V, Freschi C, Alberti A, Berchiolli R, Mosca F, et al. Electromagnetic navigation platform for endovascular surgery: how to develop sensorized catheters and guidewires. Int J Med Robot Comput Assist Surg. 2012 Sep;8(3):300-10.

13. Yoo J, Schafer S, Uneri A, Otake Y, Khanna AJ, Siewerdsen JH. An electromagnetic "Tracker-in-Table" configuration for X-ray fluoroscopy and cone-beam CT-guided surgery. Int J Comput Assist Radiol Surg. 2013 Jan;8(1):1-13.

14. Maurer CR, Fitzpatrick JM, Wang MY, Galloway RL, Maciunas RJ, Allen GS. Registration of head volume images using implantable fiducial markers. IEEE Trans Med Imaging. 1997 Aug;16(4):447-62.

15. Nijkamp J, Schermers B, Schmitz S, de Jonge S, Kuhlmann K, van der Heijden F, et al. Comparing position and orientation accuracy of different electromagnetic sensors for tracking during interventions. Int J Comput Assist Radiol Surg. 2016;1-12.

16. Tsuchida M, Yamato Y, Aoki T, Watanabe T, Koizumi N, Emura I, et al. CT-guided agar marking for localization of nonpalpable peripheral pulmonary lesions. Chest. 1999 Jul;116(1):139-43.

17. Widmann G, Stoffner R, Sieb M, Bale R. Target registration and target positioning errors in computer-assisted neurosurgery: proposal for a standardized reporting of error assessment. Int J Med Robot. 2009 Dec;5(4):355-65.

18. Kingham TP, Jayaraman S, Clements LW, Scherer MA, Stefansic JD, Jarnagin WR. Evolution of image-guided liver surgery: transition from open to laparoscopic procedures. J Gastrointest Surg. 2013 Jul 4;17(7):1274-82.

19. Lubienski A. RF Ablation of Liver Tumors. In: Mahnken AH, Wilhelm KE, Ricke J, 
editors. CT- and MR-Guided Interventions in Radiology. Second Edi. Springer; 2013. p. $213-35$.

20. Appelbaum L, Solbiati L, Sosna J, Nissenbaum Y, Greenbaum N, Goldberg SN. Evaluation of an electromagnetic image-fusion navigation system for biopsy of small lesions: assessment of accuracy in an in vivo swine model. Acad Radiol. 2013 Feb;20(2):209-17.

21. Sindram D, Simo KA, Swan RZ, Razzaque S, Niemeyer DJ, Seshadri RM, et al. Laparoscopic microwave ablation of human liver tumours using a novel threedimensional magnetic guidance system. HPB. 2015 Jan;17(1):87-93.

22. Lee MW, Kim YJ, Park SW, Yu NC, Choe WH, Kwon SY, et al. Biplane fluoroscopyguided radiofrequency ablation combined with chemoembolisation for hepatocellular carcinoma: initial experience. Br J Radiol. 2011 Aug;84(1004):691-7.

23. Pung L, Ahmad M, Mueller K, Rosenberg J, Stave C, Hwang GL, et al. The Role of Cone-Beam CT in Transcatheter Arterial Chemoembolization for Hepatocellular Carcinoma: A Systematic Review and Meta-analysis. J Vasc Interv Radiol. 2017 Mar;28(3):334-41.

24. Gu L, Liu H, Fan L, Lv Y, Cui Z, Luo Y, et al. Treatment outcomes of transcatheter arterial chemoembolization combined with local ablative therapy versus monotherapy in hepatocellular carcinoma: a meta-analysis. J Cancer Res Clin Oncol. 2014 Feb;140(2):199-210.

25. Wang X, Hu Y, Ren M, Lu X, Lu G, He S. Efficacy and Safety of Radiofrequency Ablation Combined with Transcatheter Arterial Chemoembolization for Hepatocellular Carcinomas Compared with Radiofrequency Ablation Alone: A Time-to-Event MetaAnalysis. Korean J Radiol. 2016;17(1):93-102.

26. Min JH, Lee MW, Cha DI, Jeon YH, Shin SW, Cho SK, et al. Radiofrequency ablation combined with chemoembolization for intermediate-sized $(3-5 \mathrm{~cm})$ hepatocellular carcinomas under dual guidance of biplane fluoroscopy and ultrasonography. Korean J Radiol. 2013;14(2):248-58. 